\title{
Enzymatic Production Process of Bio-based 2,5-Furandicarboxylic Acid (FDCA) from 5-Hydroxymethilfurfural (HMF)
}

\author{
Miša Mojca Cajnko, Marijan Bajić, Uroš Novak, Blaž Likozar \\ National Institute of Chemistry, Department of Catalysis and Chemical Reaction Engineering \\ Hajdrihova 19, Ljubljana, Slovenia \\ misa.cajnko@ki.si; marijan.bajic@ki.si
}

\section{Extended Abstract}

2,5-Furandicarboxylic acid (FDCA) is one of the top biomass-derived value-added chemicals. It can be produced from fructose and other sugars via formation of 5-hydroxymethilfurfural (HMF) intermediate [1]. FDCA and its derivates can be used in many fields, but the most promising application is the replacement of oil-derived tetraphtalate in the synthesis of polyethylene tetraphthalate (PET) plastics [2]. The oxidation of HMF to FDCA can proceed via two routes: (1) oxidation of the aldehyde group to form 5-hydroxymethyl-2-furoic acid (HMFA) which is then oxidized to 5-formyl-2-furoic acid (FFA) and FDCA, and (2) oxidation of the alcohol group to form 2,5-diformylfuran (DFF), followed by further oxidations to FFA and FDCA. Most of the chemical methods for FDCA production require harsh conditions, like high pressure and temperature, organic solvents and special salts, making the process environmentally unfriendly and high cost [3]. As an environmentally friendly alternative an enzymatic conversion process can be applied [4]. However, to our knowledge, only a few enzymes have been found to be active toward HMF and most of them were not able to oxidize HMF to FDCA. Thus, to achieve a full conversion with high FDCA yields, a combination of multiple enzymes is needed [5]-[7].

In order to better understand the catalytic activity of some of these enzymes we broke the oxidation of HMF to FDCA down to separate reactions to determine all the possible substrates as well as reaction products for each individual enzyme. Based on this data we then tested different combinations of enzymes in a simple one-pot reaction to determine which one gives the best results. The enzymes used in our work were commercially available horseradish peroxidase (HRP), a fungal alcohol (AO) and galactose oxidase (GO), catalase (Cat), laccase (Lac) and a lignin peroxidase (LPO) and their activity was tested against HMF, DFF, HMFA and FFA. AO, GO, HRP and LPO were active against HMF, with AO and GO producing DFF and HRP and LPO producing HMFA. All enzymes were only mildly active against DFF forming FFA and some HMF. GO, Lac, LPO and HRP were active against HMFA producing FFA and FDCA. All enzymes were also active against FFA. However, product yields obtained with these single-enzyme reactions were mostly low, the highest being $11 \%$. The exception was conversion of FFA to FDCA where some of the yields were above $80 \%$. We then prepared simple multi-enzyme reactions with HMF: AO or GO with Cat or with Cat and HRP. Combining AO and Cat notably increased the yield of DFF but decreased the yield of FFA. In the case of GO and Cat the yield of DFF also increased but to a smaller degree and with no formation of FFA. Adding HRP to each mixture increased the yield of FFA and DFF in the case of GO, but decreased the yield of DFF in the case of AO. To increase the yields further as well as obtain the final product FDCA, more enzyme combinations will have to be tested and possibly, some new enzymes considered.

\section{References}

[1] M. L. Ribeiro and U. Schuchardt, "Cooperative effect of cobalt acetylacetonate and silica in the catalytic cyclization and oxidation of fructose to 2,5-furandicarboxylic acid," Catal. Commun., vol. 4, no. 2, pp. 83-86, Feb. 2003.

[2] A. F. Sousa et al., "Biobased polyesters and other polymers from 2,5-furandicarboxylic acid: a tribute to furan excellency," Polym. Chem., vol. 6, no. 33, pp. 5961-5983, Aug. 2015.

[3] Y. Y. Gorbanev, S. K. Klitgaard, J. M. Woodley, C. H. Christensen, and A. Riisager, "Gold-Catalyzed Aerobic Oxidation of 5-Hydroxymethylfurfural in Water at Ambient Temperature," ChemSusChem, vol. 2, no. 7, pp. 672675, Jul. 2009.

[4] S. M. Thomas, R. DiCosimo, and V. Nagarajan, "Biocatalysis: applications and potentials for the chemical industry," Trends Biotechnol., vol. 20, no. 6, pp. 238-242, Jun. 2002.

[5] J. Carro et al., "5-hydroxymethylfurfural conversion by fungal aryl-alcohol oxidase and unspecific peroxygenase," 
FEBS J., vol. 282, no. 16, pp. 3218-3229, 2015.

[6] S. M. McKenna, S. Leimkühler, S. Herter, N. J. Turner, and A. J. Carnell, "Enzyme cascade reactions: synthesis of furandicarboxylic acid (FDCA) and carboxylic acids using oxidases in tandem," Green Chem., vol. 17, no. 6, pp. 3271-3275, Jun. 2015.

[7] A. Karich, S. Kleeberg, R. Ullrich, and M. Hofrichter, "Enzymatic Preparation of 2,5-Furandicarboxylic Acid (FDCA) - A Substitute of Terephthalic Acid—By the Joined Action of Three Fungal Enzymes," Microorganisms, vol. 6, no. 1, p. 5, Jan. 2018. 